\title{
LA ARQUITECTURA NEOCLÁSICA EN EL ESTADO DE MÉXICO: SUS INICIOS
}

Recibido: 15-09-2016

Aprobado: 30-10-2016

\begin{abstract}
Carlos Alfonso Ledesma Ibarra Licenciado en Historia por la UAEMéx. Estudió la maestría y el doctorado en Historia del Arte en la UNAM. Entre sus libros destacan: Las Capillas de Barrio de Malinalco (2008), El Templo y Colegio de San Ignacio de la Compañía de Jesús en Pátzcuaro (2013), Cátedra Xavier Moyssén Echeverría: México angustia de sus cristos [Coordinador] (2016). Posee diversos artículos publicados en revistas nacionales e internacionales. Profesor de Tiempo Completo de la Facultad de Humanidades de la Universidad Autónoma del Estado de México, miembro del Sistema Nacional de Investigadores (nivel 1) y profesor con perfil deseable ante la Secretaría de Educación Pública.

cledesmai@yahoo.com.mx
\end{abstract}

\author{
Carlos Alfonso Ledesma Ibarra \\ Facultad de Humanidades \\ Universidad Autónoma del Estado de México
}

\section{Resumen}

En este escrito se analiza con base en las características formales de algunos templos, el proceso de introducción del estilo neoclásico en una región del centro de México. El estilo neoclásico protegido y promovido por la Corona Española desde las academias de artes se afincó en la Nueva España desde finales del siglo XVIII. En 1783 con la fundación de la Academia de San Carlos, oficialmente, inició un proceso de transformación de los valores predominantes en la arquitectura novohispana, principalmente en la ciudad de México, pero de qué manera y cómo se fue extendiendo esta manera de construir y decorar los templos por todo el territorio del virreinato. Se eligió una región cercana a la capital del virreinato con casos precisos donde comenzó la transición al nuevo estilo y se localizaron algunos elementos comunes que me han permitido caracterizar esta región y proponer una explicación a dicho proceso.

Palabras clave: estilo, neoclásico, Estado de México, arquitectura, templos.

In this paper is analyzed based on the formal characteristics of some temples, the process of introducing the neoclassical style in a region of central Mexico. Protected and promoted by the Spanish Crown since the neoclassical art academies settled in New Spain since the late eighteenth century. In 1783 with the founding of the Academy of San Carlos, officially began a process of transformation of values prevailing in New Spain architecture, mainly in Mexico City, but how it spread this way to build and decorate temples throughout the territory of the viceroyalty. A source close to the viceregal capital in specific cases where it began the transition to the new style and some common elements that have allowed me to characterize this region and propose an explanation for this process were located region was chosen.

Keywords: style, neoclassical, State of Mexico, architecture, temples. 
Durante el periodo virreinal de México se edificaron una enorme cantidad de edificios religiosos de importante valor estético, pero desafortunadamente de la mayoría de ellos sabemos poco. Por otro lado, casi todos éstos -sobre todo los construidos entre los siglos XVII y las tres primeras cuartas partes del XVIII- se califican, indistintamente, como barrocos y son los más estudiados. En cambio, los levantados en los últimos años del siglo XVIII y la centuria decimonónica, denominados como neoclásicos, son generalmente de menor interés. No obstante, hasta ahora no se ha planteado cuál fue el proceso que llevó a las instituciones, arquitectos y mecenas de la zona central de México a cambiar los valores del "barroco" por el "neoclásico". ¿Cómo se cambió de estilo y por qué? ¿Cuáles fueron los elementos y valores estéticos que se fueron privilegiando en la zona central del actual Estado de México? ¿Cómo se constituyó en esta región un nuevo lenguaje arquitectónico?

A inicios del siglo XVIII se difundió y popularizó en el centro del virreinato $y$, especialmente, en la ciudad de México el uso del estípite ${ }^{1}$ como elemento constructivo y decorativo en retablos y fachadas, tanto civiles como religiosas. Los estípites se acompañaron de todo tipo de elementos decorativos y se convirtieron en el elemento favorito de la composición arquitectónica. Este tipo de arquitectura se denominó, por parte de los especialistas contemporáneos, como barroco estípite. Sin embargo, a mediados del mismo siglo las columnas volvieron a convertirse en el componente más importante de varias de las fachadas arquitectónicas novohispanas. Jorge Alberto Manrique designó a esta modalidad arquitectónica como neóstila. Estas modalidades del estilo barroco convivieron durante la segunda mitad del siglo en la arquitectura de la Nueva España (Manrique, 2002, 229).

El arribo de la arquitectura neoclásica, especialmente en sus inicios fue singular, pues el establecimiento de la enseñanza académica del arte en el virreinato fue a partir de la década de los ochenta del siglo XVIII (1781), por lo cual, los artistas, en especial los arquitectos, tuvieron que inclinarse ante los cambios que imponía la llegada de la Academia de San Carlos, pero la enseñanza iba a estructurar un lenguaje artístico diferente al que la tradición novohispana sugería. El problema no se redujo a la adopción de un repertorio artístico nuevo,

\footnotetext{
${ }^{1}$ Estípite: elemento troncopiramidal invertido, bien con función decorativa a manera de balaustre, o con función constructiva en lugar de una columna o pilastra.
} 
sino que iba más allá, porque la práctica de la arquitectura resulta de una compleja dinámica social donde intervienen diversos aspectos, ya que en una construcción no pueden considerarse únicamente los criterios de los arquitectos. En el proyecto y levantamiento de un nuevo edificio participan los mecenas, la disponibilidad de recursos, la reglamentación, los modelos clásicos, los vecinos (quienes muchas veces participan con su trabajo), el aspecto urbano y la tradición constructiva de una ciudad o región.

En la obra: Regionalización en el Arte. Teoría y Praxis, editado por el Instituto de Investigaciones Estéticas de la UNAM en 1992, se encuentra un escrito de la autoría del historiador José Guadalupe Victoria: "Sobre Arquitectura neoclásica en el centro de México". En dicho escrito se analizan las construcciones de las parroquias de San Martín Ocoyoacac (Estado de México), Zimapán (Estado de Hidalgo), Tulancingo (Estado de Hidalgo) y San Miguel de las Cuevas de Chalma (Estado de México). Este artículo ofrece una conclusión interesante, pues el autor afirma que en esta región al parecer se desarrolló un neoclásico que denomina "regional", pero expresa:

¿Qué fue lo que les imprimió ese carácter local? Sin poder dar una respuesta tentativa, vale la pena citar otros ejemplos no estudiados, en los que también advertimos cierta diferenciación: las iglesias de Santa Clara de Lerma, Gualupita, Capulhuac, Tenango del Valle y la Parroquia de Tenancingo (Victoria, 1992, 223).

La afirmación lanzada por el historiador Guadalupe Victoria es aún más aventurada al proponer, que esta región fue autónoma en el desarrollo del neoclásico, alejándose de los tradicionales focos de esta arquitectura: la ciudad de México y Celaya. Más aún, considero que entre estas zonas también debiera considerarse la región de Guadalajara, donde el trabajo de Manuel Tolsá y otros egresados de la Academia de San Carlos fue fundamental para la consolidación de dicho estilo en esa ciudad. No obstante, como él mismo mencionó, no propone una respuesta tentativa a esta interrogante. En este sentido, quisiera puntualizar que si bien en las primeras hipótesis de este trabajo se consideró incluir el templo de Capulhuac, posteriormente del análisis formal de algunas de sus características decidí excluirlo, pues no posee las características arquitectónicas propias del estilo neoclásico. José Guadalupe Victoria considera que con el establecimiento de la Academia de San Carlos en 1781 inició el 
cambio de valores, conceptos y formas que afectó las prácticas artísticas de todo el virreinato. Uno de los problemas más interesantes que plantea el estudio de la arquitectura novohispana, es el momento final del periodo barroco y el inicio del neoclásico.

[...] Entre 1750 y 1780 el ritmo constructivo fue muy alto en todo el virreinato y es precisamente en ese periodo cuando se erigen algunos edificios (religiosos y civiles) más significativos del barroco novohispano como el Sagrario de México, el Colegio de Tepotzotlán, Santa Prisca de Taxco entre otros. Todas esas obras fueron construidas siguiendo una tradición centenaria, tanto en su concepción como en su realización [...] (Victoria, 1992, 214).

Para este autor son dos las ciudades de mayor influencia para la mudanza de valores estéticos en la arquitectura novohispana: la ciudad de México, por la preponderancia de los arquitectos de la Academia de San Carlos y Celaya, Guanajuato, por la importancia del trabajo del polifacético artista Francisco Eduardo Tresguerras, quien influyó en la región del Bajío. Sin embargo, considera, que los historiadores del arte no han explicado cómo funcionaron dichos cambios y cómo se fueron extendiendo por el amplio territorio novohispano y si, efectivamente, fue alguna de estas ciudades la que influyó en los cambios arquitectónicos de la región centro-sur del actual Estado de México.

De acuerdo con el planteamiento del historiador José Guadalupe Victoria el estilo neoclásico se introdujo en el centro de México a partir de las construcciones del Santuario de Chalma y el templo de Ocoyoacac (separados por 56 kilómetros). El autor consideraba que ambas edificaciones modificaron, gradualmente, los valores y elementos arquitectónicos de otros templos que se construyeron o remodelaron en la región. En este caso conviene aclarar qué entiendo por "región", para este escrito, una zona que se extiende desde Ocoyoacac hasta Lerma, donde los valores y conceptos se impusieron y tuvieron una influencia mayor en algunos de los templos más representativos de sus poblaciones. No obstante, esta misma zona presenta una visible variedad climática desde la fría sierra boscosa de Ocoyoacac, hasta la cálida barranca donde se asienta Chalma. El mismo autor afirma que el Estado de México posee una gran riqueza artística aún sin estudiarse a cabalidad y que este indicio podría plantear otros estudios. 
El espacio donde se realizó esta investigación fue el centro - sur del actual Estado de México, pues esta zona presenta una unidad histórica, cultural y de cercanía geográfica que permite suponer rutas de intercambio comercial entre estas poblaciones. Además, de las peregrinaciones de éstas al Santuario de Chalma. En un sentido más específico consideré los templos de San Martín en Ocoyoacac

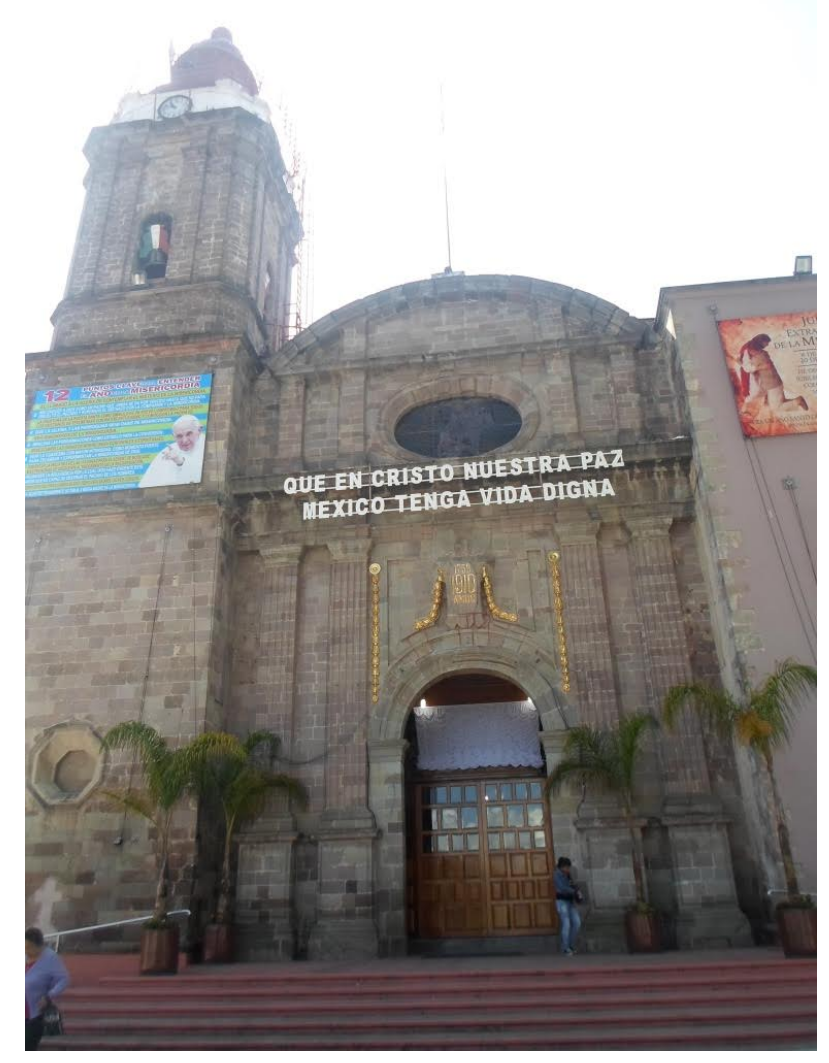

Imagen 1. Fachada del Templo de San Martín

Ocoyoacac, Estado de México. Junio de 2016.

Fotografía: Carlos Alfonso Ledesma Ibarra.
[Imagen 1], El Santuario del Señor de Chalma [Imagen 2], la parroquia de Santa Clara en Lerma [Imagen 3], la parroquia de Santa María de Guadalupe [Imagen 4], en el poblado de Guadalupe Yanhuitlalpan, municipio de Santiago Tianguistenco, el templo de la Asunción en Tenango del Valle [Imagen 5] y la parroquia de San Francisco de Asís en Tenancingo [Imagen 6]. No obstante, reconocí desde el primer acercamiento a estas construcciones, diferentes maneras de aplicar los elementos reconocidos como neoclásicos en la arquitectura. Por otro lado, ninguno de estos edificios se había estudiado bajo el cuidado de conceptos específicos y tampoco se había investigado sobre la posible relación formal y arquitectónica que pudieron haber tenido con otros edificios. La mayoría fueron construidos, reconstruidos o remodelados, casi de forma simultánea, a finales del siglo XVIII y las primeras tres décadas del siglo XIX.

Indudablemente, que las características arquitectónicas y constructivas de los templos antes mencionados, guardan una relación que aún no ha sido explicada bajo conceptos específicos. Asimismo, con este estudio se contribuye a la necesidad de conocer 
para resguardar nuestro patrimonio arquitectónico. Es un error común creer que los edificios construidos durante el siglo XIX no poseen el mismo valor histórico y estético de otros más antiguos. Tal vez sea este enorme prejuicio el que llevó al Estado de México a perder una importante cantidad de patrimonio proveniente de la centuria decimonónica. Igualmente, debe mencionarse que a simple vista es evidente la existencia de características neoclásicas específicas que se fueron constituyendo en un lenguaje arquitectónico predominante y que había permanecido sin ser estudiado en la región mencionada.

Para el historiador Israel Katzman el periodo comprendido entre 1790 y 1810 fue uno de los de mayor actividad constructiva en la Nueva España. En los siguientes diez años, debido a la Guerra por la Independencia, dicha actividad disminuyó significativamente, para reactivarse, nuevamente, hasta 1820 y mantenerse estable durante los siguientes diez años (Katzman, 1993, 9). Para el caso de esta zona tengo la certeza documental y formal de la remodelación del templo de Ocoyoacac y, especialmente de su fachada desde finales del siglo XVIII y principios de la siguiente centuria. En esta primera etapa del neoclásico novohispano se observa, en palabras del propio Katzman, un clasicismo plano, "embarrado", bidimensional (Katzman, 1993, 9). En este caso parecen ubicarse las fachadas de Ocoyoacac y de Santa Clara de Lerma.

Por otro lado, debiera subrayarse que la fachada del Santuario de Nuestro Señor de Chalma no es contemporáneo de Ocoyoacac. A pesar de lo significativo que debió ser el nombramiento de éste bajo la protección del rey, designado como Real Convento y Santuario bajo su protección en 1783, se conservó la fachada construida, tal vez en las remodelaciones de mediados del siglo XVIII, por lo menos hasta principios del siglo XIX, pues para sostener esta información contamos con la publicación de Joaquín Sardó: Relación histórica y moral de la portentosa imagen de N. Sr. Jesucristo crucificado aparecida en Chalma, impreso por la casa de Arizpe en 1810, donde todavía se describe una fachada barroca muy diferente a la que ha llegado hasta nuestros días (Katzman, 1993, 9).

En los templos analizados en este estudio: San Martín en Ocoyoacac, Santa Clara en Lerma, La Asunción en Tenango del Valle, San Francisco en Tenancingo, Santa María de Guadalupe en Santiago Tianguistenco y el Santuario de Chalma se observa el desarrollo de un estilo propio en la decoración de las fachadas de los edificios 
construidos en la segunda mitad del siglo XVIII, el cual privilegió la inclusión de decoración fitomorfa en los elementos arquitectónicos y argamasa como el material más usado para la decoración de las fachadas. Este tipo de elementos sería desplazado o combinado a partir de la introducción de algunas ideas y modelos propios de la arquitectura neoclásica. No obstante, dichos elementos pueden reconocerse en el capitel de las columnas de "Gualupita" (Con este nombre se designa tradicionalmente al templo de Santa María de Guadalupe, en el barrio de Guadalupe Yancuitlalpan en Santiago Tianguistenco) o en el resplandor del remate de la fachada de Santa Clara en Lerma.

Pero qué modelo del arte neoclásico siguieron estos edificios. Primeramente, puedo afirmar que formalmente fueron más cercanos a la arquitectura practicada en la ciudad de México. Específicamente, su primera influencia proviene de dos obras del propio José Antonio González Velázquez, quien estuvo a cargo de la construcción del templo del Santo Desierto de los Carmelitas en Tenancingo; sin embargo, esta obra no tuvo gran difusión debido a su permanencia dentro de un edificio dedicado al aislamiento. No obstante, el mismo arquitecto estuvo encargado del proceso de remodelación de la Parroquia de San Martín en Ocoyoacac. Esta vez, su influencia fue decisiva para las futuras construcciones de esta región. Sabemos por documentos y su propia obra que Antonio González Velázquez fue un seguidor puntual de los ideales académicos de la arquitectura clásica. Prefirió, entre los órdenes de la arquitectura, el dórico con sus fustes estriados y capiteles sin molduras. Otra característica visible en varios de estos edificios, pero inicialmente en la parroquia de San Martín en Ocoyoacac es la monumentalidad de los elementos clásicos, propios del trabajo arquitectónico de dicho autor.

No obstante la conocida severidad de dicho maestro, la aplicación del estilo neoclásico en estos edificios, queda lejos de la rigurosidad que se pretendía en la Academia de San Carlos. Por el contrario, en todos los casos se encuentran elementos no propios de la arquitectura neoclásica e interpretaciones en los elementos clásicos alejadas de las proporciones registradas en cualquiera de los tratados seguidos por los arquitectos academicistas. Más aún, tendría que dejar fuera de esta consideración a la fachada del Templo de la Asunción de Tenango del Valle, la cual es más cercana a la modalidad barroca del neóstilo, pues conserva perfiles mixtilíneos, varias esculturas en nichos 
y el tratamiento de sus semicolumnas compuestas es lejana a la corrección neoclásica. Sin embargo, debiera mencionarse que en esta población se localizan dos ejemplos posteriores, de finales del siglo XIX, de un neoclásico académico: el templo de Nuestra Señora de Guadalupe y la fachada de la capilla del Calvario, quizás, el primero de estos edificios cercano a la fachada de Chalma, pues posiblemente el artífice de Tenango haya participado en la proyección de la fachada del Santuario.

Sin embargo, la mayoría de los edificios aquí referidos no se parecen a la bidimensionalidad del templo de Ocoyoacac practicada, originalmente, en la fachada de Santa Clara de Lerma. La mayor parte de éstos posee una mayor espontaneidad en la ejecución de los estilos y la integración de elementos no contemplados en la arquitectura clásica. Además, la tridimensionalidad es evidente en "Gualupita", Tenancingo y Chalma, aunque de estos ejemplos sólo los dos últimos parecieran procurar

Imagen 2. Fachada del Santuario de Chalma, Estado de México. Junio de 2016. Fotografía: Carlos Alfonso Ledesma Ibarra.

un repertorio cercano a los tratados clásicos. Más aún, en el caso del Santuario de Chalma es mayor su proximidad a los tratados de arquitectura como es evidente, pero sin llegar a su aplicación rigurosa.

Otra característica a considerar se tiene en la existencia de algunas fachadas laterales en los templos de San Martín en Ocoyocac, Santa Clara en Lerma y San Francisco de Asís en Tenancingo. En los primeros dos casos la puerta de acceso es un arco de medio punto, el cual se encuentra enmarcado por pilastras dóricas. Éstas se elevan sobre plintos, en el segundo edificio éstos presentan motivos fitomorfos modelados en argamasa. En el primero de los casos los fustes tienen almohadillados y en el segundo se observa una 
profunda acanaladura. En los dos casos sostienen un entablamento que la clave de la puerta se abulta para sostener en el caso de Ocoyoacac una talla del Sagrado Corazón de Jesús con un cordero en medio. En Santa Clara ahí se ubica un nicho, actualmente, vacío. En ambas composiciones en las esquinas del entablamento se levantan pináculos, mayores y de mejor talla en el edificio de Ocoyoacac. La fachada lateral de Tenancingo es un arco de medio punto, enmarcado entre un par de pilastras jónicas lisas y sobre éstas se sostiene un entablamento que sirve de base a una ventana de medio círculo.

El orden predominante en las columnas, semicolumnas y pilastras de esta serie de edificios es el dórico: Ocoyoacac, Chalma y Tenancingo lo presentan. Le sigue el toscano que se observa en las semicolumnas de la Asunción en Tenango y la fachada de Santa Clara de Lerma.

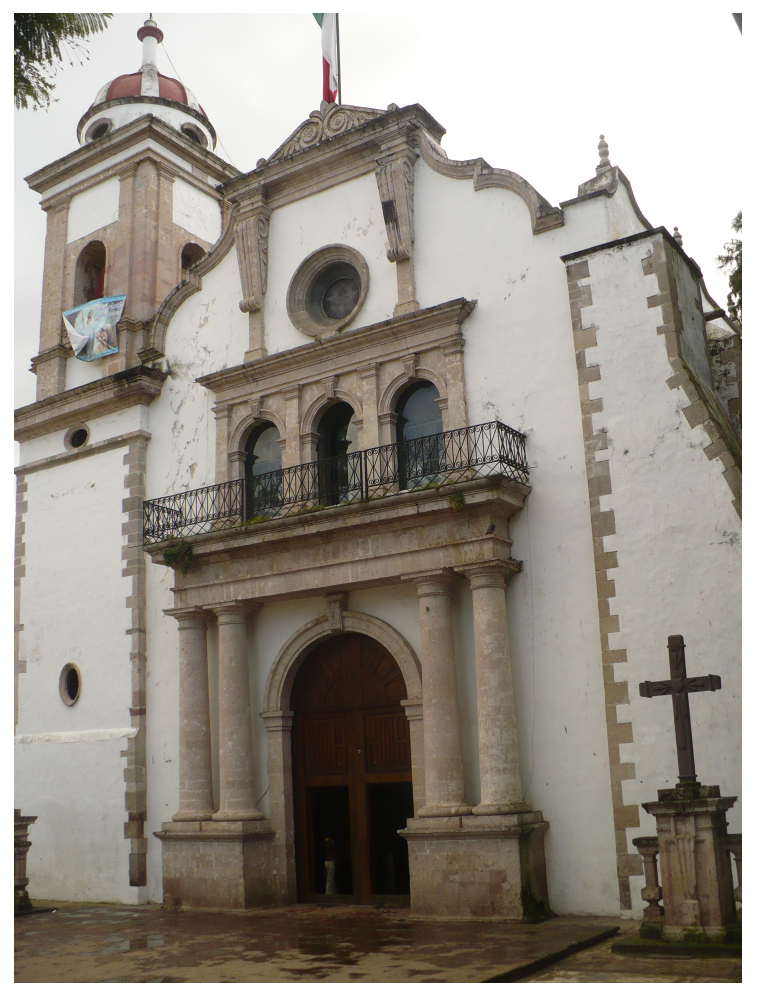

Imagen 3. Fachada del Templo de Santa Clara, Lerma, Estado de México. Junio de 2016. Fotografía: Carlos Alfonso Ledesma

' Finalmente, mención aparte merece el estilo compuesto practicado en el templo de "Gualupita" con todos esos elementos singulares y ajenos al estilo neoclásico. En el mismo sentido, es de subrayarse que en estos edificios no se practicó la combinación de órdenes clásicos. El orden practicado en el primer cuerpo era seguido en el segundo y hasta el remate, pero se disminuía el tamaño y, en ocasiones, se pasaba de la semicolumna o columna a la pilastra como en los casos de Lerma y Tenancingo.

Resulta evidente que tampoco existe regularidad en la forma de estas fachadas, mientras algunas aún son de forma mixtilínea como Tenango del Valle y Tenancingo; otros son arcos lunetos: Ocoyoacac y "Gualupita". Nuevamente, la más cercana a los órdenes clásicos es la forma rectangular de la fachada del Santuario 
de Chalma más parecida a la fachada del Templo de Guadalupe de Tenango del Valle. En cuanto al remate de estas formas en los templos de Chalma y "Gualupita" se observa el uso de una balaustrada.

El remate de las torres campanario es otro elemento propio de las obras estudiadas. La forma de campana, característica del remate de las torres campanario de la Catedral Metropolitana proyectadas en las postrimerías del siglo $\mathrm{XVIII,} \mathrm{por} \mathrm{el} \mathrm{arquitecto}$ novohispano, José Cosme Damián Ortiz

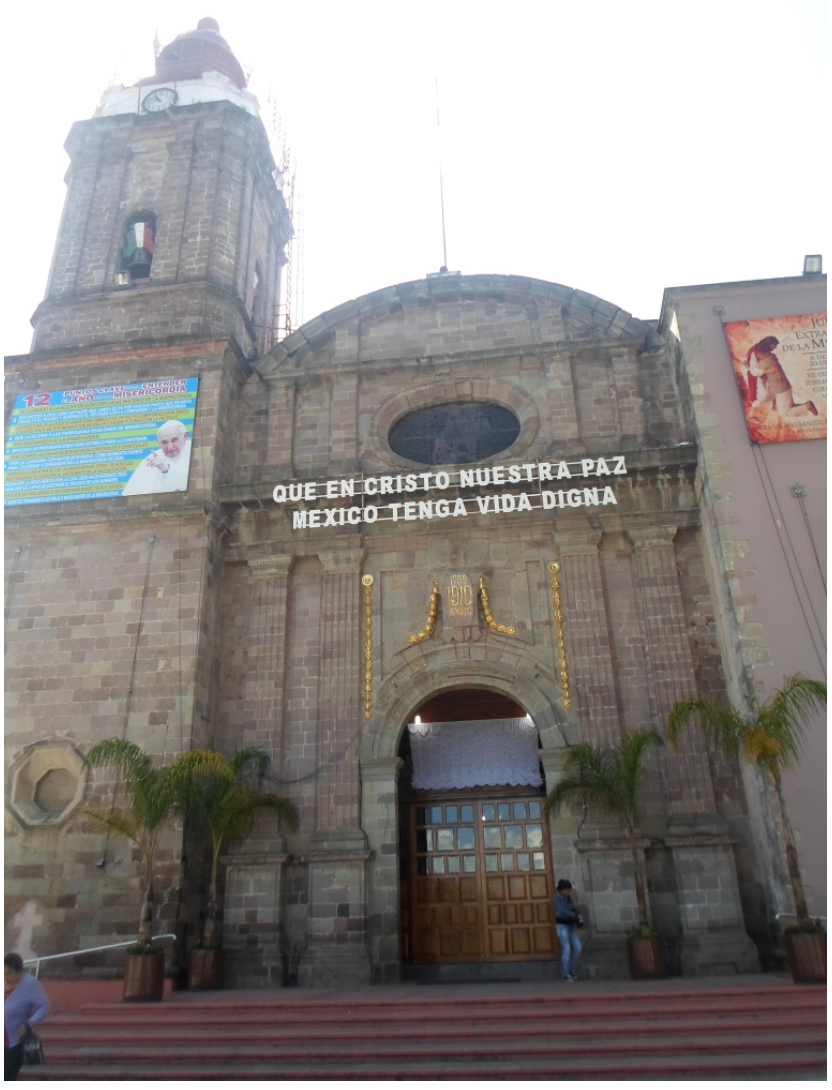
de Castro se observan en San Martín Ocoyoacac, el Santuario de Chalma, Santa Clara en Lerma y el templo de "Gualupita". Es decir, que templos que comenzaron la construcción de este elemento en los primeros años del siglo XIX, en San Martín Ocoyoacac, y otros que la

Imagen 4. Fachada del Templo de Guadalupe, Santiago Tianguistenco, Estado de México. Junio de 2016. Fotografía: Carlos Alfonso Ledesma Ibarra.

concluyeron hasta mediados del siglo $X X$, como el templo de "Gualupita", repitieron esta forma de remate. Muchas veces, el último elemento que se integró. Más aún, resulta interesante que en los casos restantes de los templos de la Asunción en Tenango y San Francisco de Asís en Tenancingo la base del remate de la torre sea redonda.

En cuatro de estos edificios la nave es de cruz latina y sólo en un par Tenango y Tenancingo es de cañón corrido, pues posiblemente, conservaron naves anteriores. En los cruceros de las naves se erigieron cúpulas de base octagonal o circular pero siempre con 
ventanas en el tambor. Más aún, debe subrayarse que incluso en el caso de Tenango en el presbiterio se elevó una cúpula que le otorga mayor luminosidad. Esta característica es común en todos los edificios que no escatiman en amplios ventanales a lo largo de las naves, en el coro, en los tambores de las cúpulas, en los brazos del crucero, en las capillas o en el presbiterio. Sus formas son variadas, desde el tradicional óculo abocinado y el medio círculo en Tenancingo, amplios arcos recortados y arcos de medio punto en Chalma, una ventana ovalada en el coro de Ocoyoacac, el hexágono y el rectángulo en Lerma.

En todos estos casos se debe explicar el concepto de estilo neoclásico como producto del contexto histórico y los templos como resultado de problemáticas específicas que permiten reconocer y entender las variables que pueden contenerse en dicho estilo, a pesar de que éste, desde el academicismo procuró cierta uniformidad en la aplicación estricta de los cánones y modelos provenientes de los tratados de arquitectura. Sin embargo, esto se somete a un contexto de pequeñas poblaciones rurales a finales del siglo XVIII y principios del XIX que no pudieron eludir la tradición en la arquitectura y la decoración.

Todos estos templos se construyeron en el contexto de pequeñas poblaciones. Es decir, no estuvieron en lugares de demografía importante. De esta manera, también se muestra la forma en que se expandieron los valores de la arquitectura clasicista desde la Academia de San Carlos hasta las poblaciones del interior del virreinato y, posteriormente, México. De ahí la importancia de definir, primeramente, el concepto de estilo como formas y valores estéticos dinámicos, cambiantes y adaptables.

La arquitectura neoclásica en la Nueva España tuvo una introducción que supo combinar la tradición de la historia de la arquitectura novohispana y los cánones, conceptos y valores propios de la arquitectura neoclásica europea. Los arquitectos novohispanos mudaron, gradualmente, sus valores durante la segunda mitad del siglo XVIII, abandonaron el uso del estípite y prefirieron utilizar las columnas como el elemento más importante en las composiciones de las portadas. En buena parte del territorio del centro del virreinato, nuevamente, fue el elemento central de las estructuras en las fachadas de la arquitectura civil y religiosa, acompañadas de tallas y con ventanas mixtilíneas o lobuladas. En este sentido, pueden observarse 
varios ejemplos en la región estudiada, entre éstos destacan: el templo de Santa María del Buen Suceso localizado en Santiago Tianguistenco, al parecer impulsado en su primera etapa constructiva por el rico minero José de Borda (mecenas de la construcción de la parroquia de Taxco) y la parroquia de San Bartolomé Apóstol en Capulhuac. El templo, primeramente aludido, presenta en su fachada una destacada selección de columnas que lo relacionan con el templo de Santa Prisca en Taxco. En cambio, en el caso de San Bartolomé en Capulhuac su fachada es muy parecida a la parroquia de Santiago en Chalco, obra del reconocido arquitecto novohispano Ignacio de Castera y construida entre 1780 y 1795.

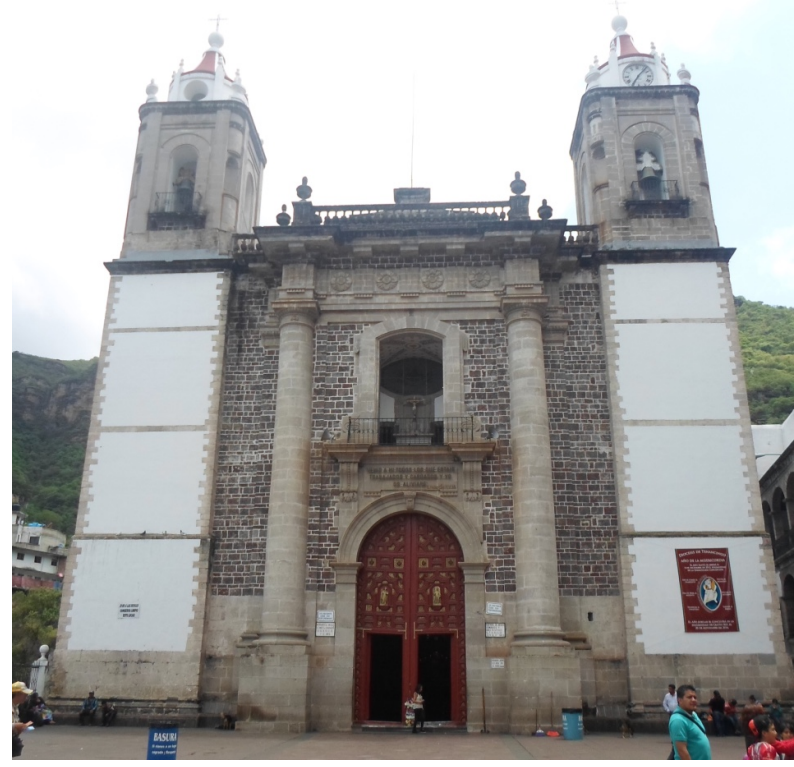

Más aún, después de esta investigación debieran considerarse entre estos edificios neóstilos al templo de Nuestra Señora de la Asunción en Tenango del Valle y la capilla de Santa María Magdalena, Ocotitlán, en el cercano municipio de Metepec, pues ambos presentan características similares en la composición de su fachada. Si bien es cierto que coincido con Jorge Alberto Manrique, al señalar que el neóstilo

Imagen 5. Fachada del Templo de la Asunción, Tenango del Valle, Estado de México. Junio de 2016. Fotografía: Carlos Alfonso Ledesma Ibarra. no fue una causa del establecimiento y el éxito del neoclásico, indudablemente, fue su

antecedente. Es decir, los arquitectos, talladores y clientes que vivieron y trabajaron estos años de transición y establecimiento de los valores academicistas eran poseedores de una tradición de trabajo que se modificó para incorporarse a la expansión del estilo neoclásico, pero sin borrar los conocimientos que ya poseían. En otras palabras, las supuestas incorrecciones que los puristas encuentran en esta primera etapa de la arquitectura neoclásica se debe, 
principalmente, a una tradición regional en la forma de trabajar que también se mantuvo en el gusto de la población.

No obstante, conviene recordar que el clasicismo y sus valores no eran desconocidos para los arquitectos novohispanos, ni siquiera en los periodos donde las construcciones más frecuentes recurrieron a una cargada decoración de las fachadas y los retablos. Esta noción permitió explicar que el cambió de estilo fue a partir de un movimiento que cobró mayor fuerza debido a la fundación de la Academia de San Carlos, pero ésta no fue la única causa, puesto que existieron arquitectos llegados de la península ibérica y Europa ya con el germen de estas ideas. Asimismo, debe considerarse el tráfico y comercio de libros, pues se volvieron cada vez más comunes algunas publicaciones que los propios arquitectos locales se preocuparon por difundir y cultivar entre el público en general y sus colegas para impulsar los conceptos y valores propios de la arquitectura neoclásica.

De esta manera, el primer edificio neoclásico de esta región fue el templo de San Martín en Ocoyoacac, el cual fue proyectado por el director del ramo de arquitectura de la Academia de San Carlos, Antonio González Velázquez, pero con la coautoría de alguno de sus ayudantes, posiblemente José Gutiérrez. Sin embargo, el neoclásico que se observa en dicho edificio es lejano de los cánones más estrictos sobre el uso de los órdenes clásicos. No obstante, dicho orden se aleja aún más en los otros ejemplos de edificios cercanos como Santa Clara en Lerma y "Gualupita" en Santiago Tianguistenco. En el primero de los casos se incluyeron en la fachada modelados en argamasa. En el segundo se incluyeron elementos externos y extraños a los órdenes clásicos. Más aún, el templo de San Martín tampoco parece más cercano a lo realizado en las fachadas de los edificios de Tenancingo y Chalma, pues ambos parecen posteriores en sus formas, ejecución y composición.

El neoclásico de estos templos es más cercano, formalmente, al practicado en la ciudad de México, pero donde se distingue la tradición constructiva y decorativa de la región. Por otro lado, pareciera que nunca se ajustó formalmente a los cánones propios de la corrección neoclásica, pero logró construir un lenguaje acorde con las necesidades de sus arquitectos y población, donde se combinaba el uso de los conceptos neoclásicos, pero sin abandonar completamente elementos decorativos como los modelados en 
argamasa o la inclusión de algunos elementos que les permitieran distinguirse de los otros templos como los remates mixtilíneos en la Asunción de Tenango del Valle y San Francisco en Tenancingo. Este par de ejemplos son muestra de la transgresión a la línea recta, uno de los valores fundamentales del neoclásico en otros lugares.

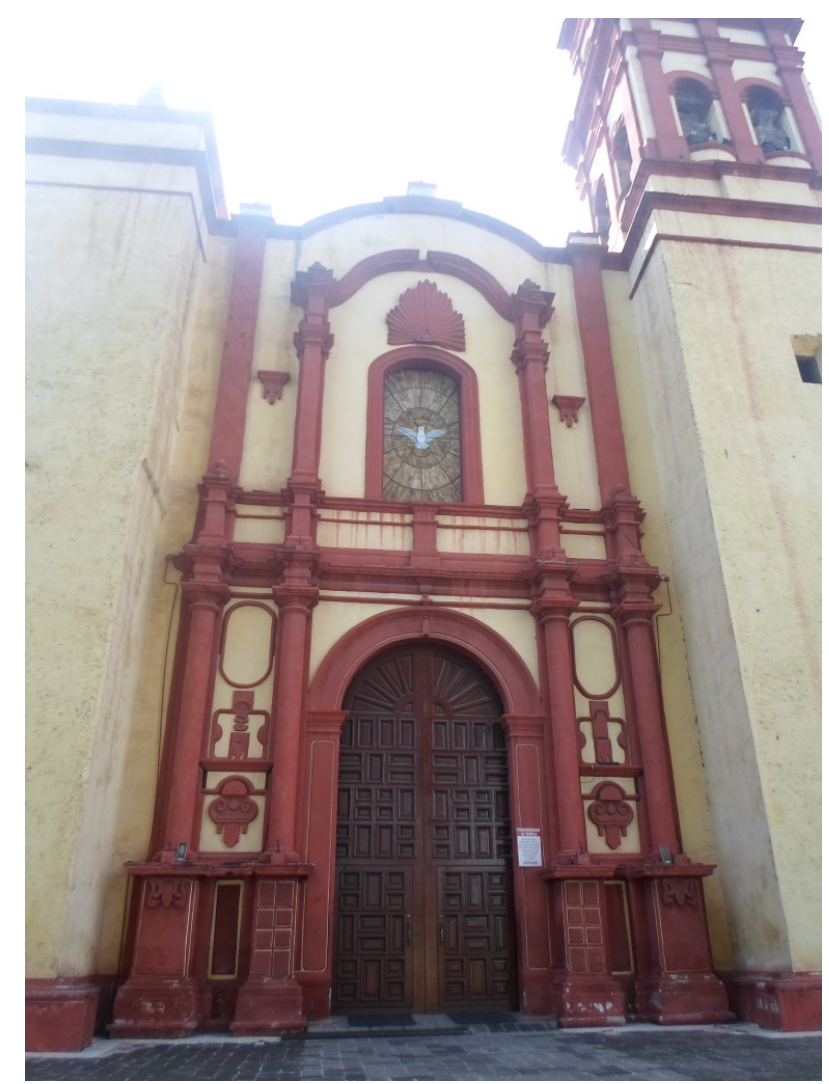

En otras palabras, en la mayoría de los edificios: Santa Clara de Lerma, "Gualupita" y San Francisco en Tenancingo se permitieron la incorporación de elementos constructivos y decorativos que no pertenecen a los órdenes clásicos, pero a estos templos sería imposible negarles su inclusión en la práctica de la arquitectura neoclásica, pero en estos casos cercanos con una tradición constructiva y decorativa que procuraba la decoración del tallado Imagen 6. Fachada del Templo de San Francisco, Tenancingo, Estado de México. Junio de 2010. Fotografía: Carlos Alfonso Ledesma Ibarra. con argamasa o la posibilidad de incluir balaustradas, tallas,

florones y jarrones. Es decir, no se estableció un patrón preciso o características comunes recurrentes que permitan identificar el neoclásico de esta región.

Los órdenes clásicos procurados por la arquitectura academicista no se practicaron con rigurosidad en estos edificios, salvo, quizás, en los templos de Ocoyoacac y Chalma. Si bien es cierto, que junto a este par de fachadas que procuraron el orden dórico deben sumarse a las columnas y pilastras dóricas del templo de San Francisco de Asís en Tenancingo, pero donde al mismo tiempo se incluyen volutas en el remate de la fachada. En cambio, los templos de Santa Clara en Lerma y La Asunción de Tenango de Valle presentan fachadas donde las pilastras y semicolumnas toscanas son el elemento fundamental de 
su composición. No obstante, conviene recordar que la fachada primigenia de Santa Clara se encuentra detrás de la que actualmente se observa. Finalmente, mención aparte merece la composición de la fachada de "Gualupita", donde el orden compuesto de la columnas se combina con florones de todos tamaños y esculturas. Se observan en estos edificios elementos clásicos que se combinan con tallas y elementos ajenos a dicha tradición, pero que parecieran imprescindibles en la decoración de los templos de esta región.

Indudablemente que esta investigación sólo es el primer paso de muchos para explicar la aparición, el funcionamiento y la expansión del estilo neoclásico en la arquitectura religiosa en el actual Estado de México, pues resulta evidente, el gusto que tuvieron en esta zona por este estilo difundido desde la academia de San Carlos. En dicho sentido resultan sobresalientes los casos de Tenango del Valle y Lerma. El primero de estos poblados con las construcciones posteriores del Templo de Santa María de Guadalupe y la fachada de la capilla del Calvario, ambos, son ejemplos de un repertorio arquitectónico más tardío, pero que procura un seguimiento más puntual de los órdenes clásicos, en ambos casos el jónico.

Más aún, queda pendiente aclarar la relación entre las fachadas del templo de Guadalupe en Tenango del Valle y el Santuario de Chalma, pues la participación del arquitecto José María Gómez, quien en 1880 construía el templo de Guadalupe y al parecer participó, anteriormente, en algunas reconstrucciones no especificadas en el Santuario de Chalma. Éste falleció en 1889 y lo enterraron en el templo de Guadalupe (Katzman, 1993, 357). Es probable, que éste sea el artífice de la fachada del Santuario de Chalma y la capilla del Calvario en Tenango del Valle. Resulta interesante, este proceso donde el objetivo de incorporar las prácticas arquitectónicas novohispanas al neoclásico, reconocido como el estilo correcto y moderno desde el último tercio del siglo XVIII fue adoptado, paulatinamente, por los arquitectos y la población de zonas rurales pero incorporando, al nuevo estilo, sus tradiciones constructivas y decorativas.

\section{BIBLIOGRAFÍA}

Brown, Thomas A. (1976) La Academia de San Carlos en la Nueva España. Fundación y organización. México: SEP - Setentas. 
Estrada, Genaro. (1935) Algunos papeles para la historia de las Bellas Artes en México. México: Academia de Bellas Artes de San Carlos.

Fernández, Justino. (1989) El arte del siglo XIX en México. 3a Edición. México: Universidad Nacional Autónoma de México.

Flores Flores, Óscar [Coordinador]. (2014) El clasicismo en la época de Pedro José Márquez (1741 - 1820), México: UNAM - IIE - Real Academia de Bellas Artes de San Fernando.

Katzman, Israel. (1993) Arquitectura del siglo XIX en México. 2a edición. México: Trillas.

Katzman, Israel. (2002) Arquitectura religiosa en México (1780 - 1830). México: FCE - UNAM.

Manrique, Jorge Alberto. (2002) Una visión del Arte y la Historia en México. t. IIII. México: UNAM.

Mendiola Quezada. (1993) Vicente, Arquitectura del Estado de México: en los siglos XVII, XVIII y XIX. Toluca: Instituto Mexiquense de Cultura.

Obregón, Gonzalo. (1953) "El Real Convento y Santuario de San Miguel de Chalma" en Estudios históricos americanos. México: El Colegio de México.

Ortiz Macedo, Luis. (2012) El arte neoclásico en México. México: Miguel Ángel Porrúa, La Serie Historia.

Sahagún de Arévalo, (1949) Juan Francisco, Gacetas de México, v. II. México: Secretaría de Educación Pública.

Sardó, Joaquín Fray. (1810) Relación histórica y moral de la portentosa imagen de N. Sr. Jesucristo crucificado aparecida en Chalma. México: Impresa en Casa Arizpe. 
Sicardo, José. (1683) Interrogatorio de la vida y virtudes del venerable hermano Fr. Bartholomé de Jesús María. México: Impreso por Juan Ribera.

Utrilla Hernández, Alejandra. (2004) Arquitectura religiosa del siglo XIX: catálogo de planos del acervo de la Academia de San Carlos. México: UNAM-Academia de San Carlos. 